\title{
Corrigendum: Novel DNA binding domain and genetic regulation model of Bacillus subtilis transition state regulator AbrB
}

\section{Jeffrey L Vaughn, Victoria Feher, Stephen Naylor, Mark A Strauch \& John Cavanagh}

Nat. Struct. Biol. 7, 1139-1146 (2000).

Based on a recent bioinformatics analysis, we have refined the dimeric interface of the N-terminal domain of the Bacillus subtilis transition state regulator AbrB. Comprehensive sets of $\sim 300$ unambiguous distance constraints (NOEs) and hydrogen exchange data have been collected that now accurately define this interface. There is an additional $\beta$-structure formed between two interlocking subunits of the homodimer. The coordinates of the revised structure and relevant information are now available in the Protein Data Bank (accession code 1Z0R). The original structure described in the manuscript (accession code 1EKT) is now obsolete. The modified AbrB N-terminal domain structure is more consistent with biophysical

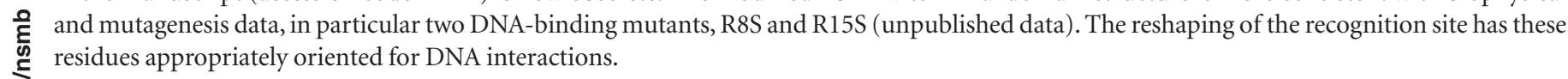

